\title{
Ausschöpfung der vollen Leistungsfähigkeit von Sortiersystemen durch neue Einschleustechnik
}

\author{
Volker Sadowsky \\ Kai F. Semrau \\ Lehrstuhl für Förder- und Lagerwesen FLW, \\ Technische Universität Dortmund
}

\begin{abstract}
D ie Ausschöpfung der vollen Leistungsfähigkeit von Stückgut-Sortiersystemen bedingt eine stetige $\mathrm{Zu}$ förderung der Stückgüter über die Einschleusungen zum Sorter. Für Sortiersysteme mit nur einer Einschleusung trifft dies in besonderer Weise zu, da in diesem Fall die maximale Durchsatzleistung des Sorters direkt durch die maximale Durchsatzleistung der Einschleusung begrenzt wird. Im Rahmen dieses Artikels werden unterschiedliche Gestaltungsmöglichkeiten für die Einschleusung am Drehsorter vorgestellt, mit denen unterschiedliche Leistungsniveaus erreicht werden können. Im Vordergrund steht dabei eine Fördertechnik zur Synchronisation der Stückgüter mit dem Sorter, mit der eine dynamische Einschleusung von bis zu 6000 Stückgütern pro Stunde realisiert werden kann. Eine aufwändige Regelung mehrerer Einschleusbänder entfällt dabei.
\end{abstract}

[Schlüsselwörter: Drehsorter, Einschleusung, Sorter, Sortiersystem, SpinSorter]

\section{Einleitung}

Die Einschleusung von Stückgütern in Sortiersystemen ist eine anspruchsvolle steuerungstechnische Aufgabe und trägt wesentlich zur Ausschöpfung der vollen Leistungsfähigkeit von Sortiersystemen bei. Dies trifft insbesondere auf solche Sortiersysteme zu, bei denen baubedingt nur eine Einschleusung realisiert werden kann. In diesen Fällen resultiert die effektive Leistung des Sorters direkt aus der Einschleusleistung. Bei Sortern mit einer Einschleusung hat die Einschleusleistung somit einen entscheidenden Einfluss auf die Gesamtleistung des Stückgut-Sortiersystems und damit auch auf die Leistung des gesamten Logistiksystems.

Stückgut-Sortiersysteme werden eingesetzt, um z.B. in Distributions- oder Logistikzentren die Sortierung bzw. Verteilung der Güter zu übernehmen. Sie sorgen für die Verteilung der in ungeordneter Reihenfolge ankommenden Güter aufgrund vorgegebener Unterscheidungsmerkmale auf mehrere Ziele [VDI3619]. Das zentrale Element eines Sortiersystems ist der Sorter [JH06]. Er besteht neben dem Verteilförderer, der die Beförderung von Gütern übernimmt, aus Einschleusungen, die für die Übergabe der Stückgüter von der Stetigfördertechnik auf den Ver- teilförderer zuständig sind und aus Endstellen, in die die Güter verteilt bzw. sortiert werden (Abb.1).

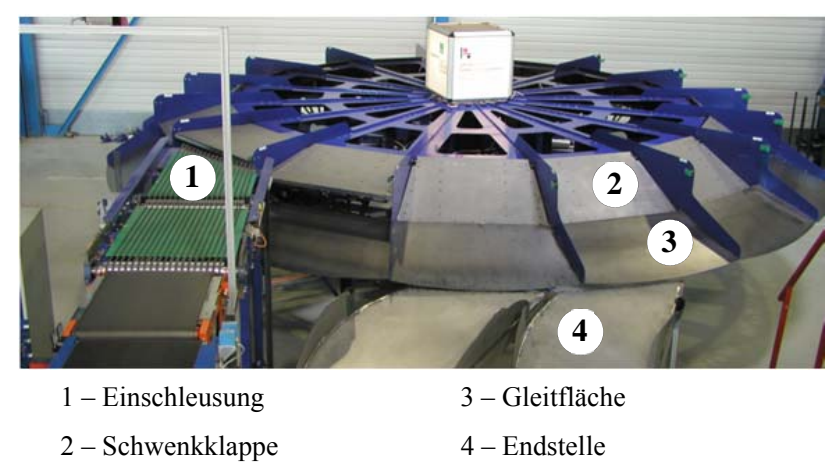

Abbildung 1. Drehsorter Prototyp

Sorter werden in den verschiedensten Branchen eingesetzt und unterliegen daher unterschiedlichsten Leistungsanforderungen. Zudem müssen die verschiedensten Artikel sicher gehandhabt werden. Aus diesem Grund existieren am Markt viele unterschiedlich gestaltete Sorter mit einer großen Leistungsspannbreite. $\mathrm{Zu}$ unterscheiden sind hierbei im Wesentlichen zwei Leistungsklassen. Im niedrigen Leistungsbereich bis 5000 Stk./h werden bevorzugt Pusher und Transfersysteme eingesetzt. Kippschalensorter, Quergurtsorter und Schiebeschuhsorter dominieren im Leistungsbereich oberhalb von 5000 Stk./h, bei dem von Hochleistungssortern gesprochen wird [JH06].

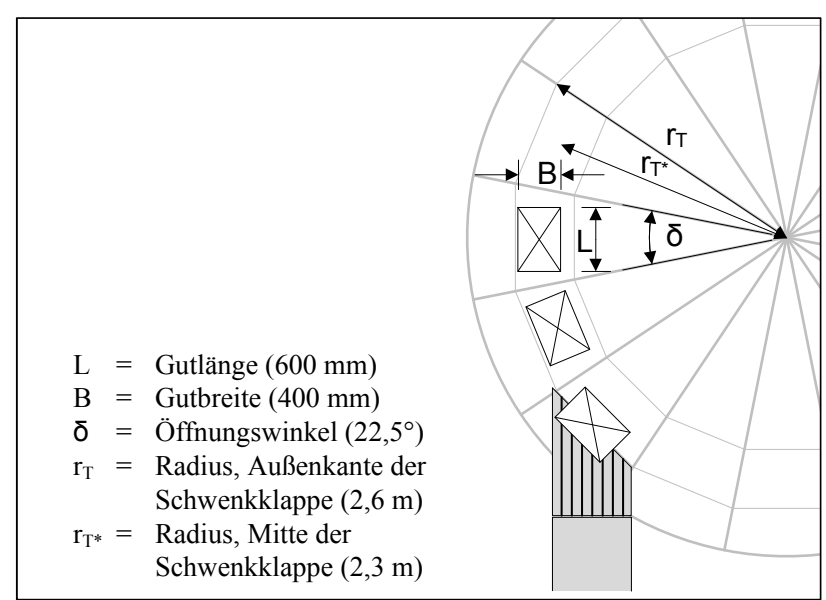

Abbildung 2.

Geometrische Verhältnisse am Prototypen (Draufsicht) 
Der vom Lehrstuhl für Förder- und Lagerwesen FLW der Technischen Universität Dortmund und dem Fraunhofer-Institut für Materialfluss und Logistik IML entwickelte Drehsorter (Abb.1) wurde mit dem Ziel niedriger Investitions- und Betriebskosten für den mittleren Leistungsbereich konzipiert. Der am FLW vorhandene Prototyp des Drehsorters ist für das Sortieren und Verteilen von
Stückgütern mit einer Länge von bis zu $600 \mathrm{~mm}$ Länge geeignet (Abb.2). Am Prototyp wurden verschiedene Einschleusungen und verschiedenartige Ausschleusungen realisiert, um das Bewegungsverhalten der Stückgüter während der Einschleusung auf den Sorter und der Ausschleusung über die mitrotierenden Gleitflächen zu untersuchen.

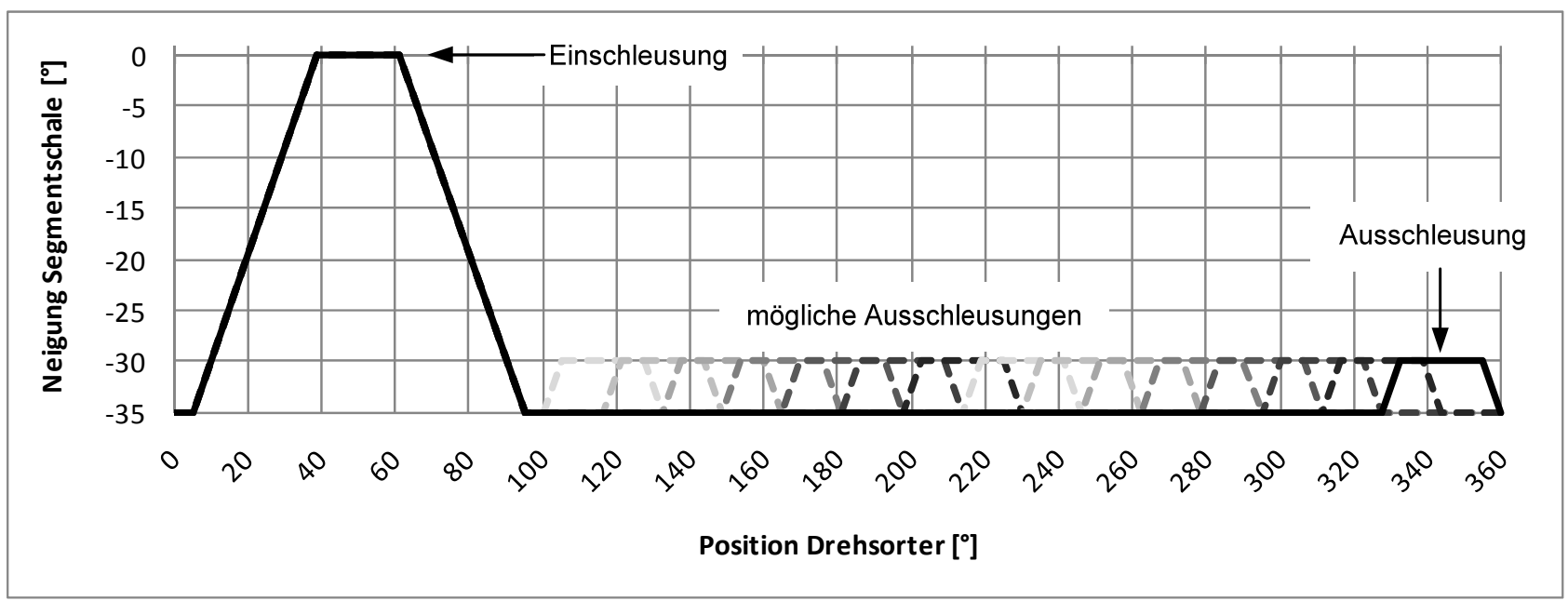

Abbildung 3. Neigung einer Segmentschale während einer Drehsorterumdrehung

Das Kernelement des Drehsorters ist der kegelstumpfförmige Drehteller, der über seine Drehbewegung Stückgüter in seinen 16 Schwenkklappen befördern und auf die außen angeordneten Endstellen verteilen kann (Abb.4a). Außerhalb der Schwenkklappen befindet sich eine mitrotierende Gleitfläche, die Bestandteil des Drehtellers ist und über die die Güter bei der Ausschleusung gleiten, bevor sie die Endstellen erreichen. Die Stückgüter
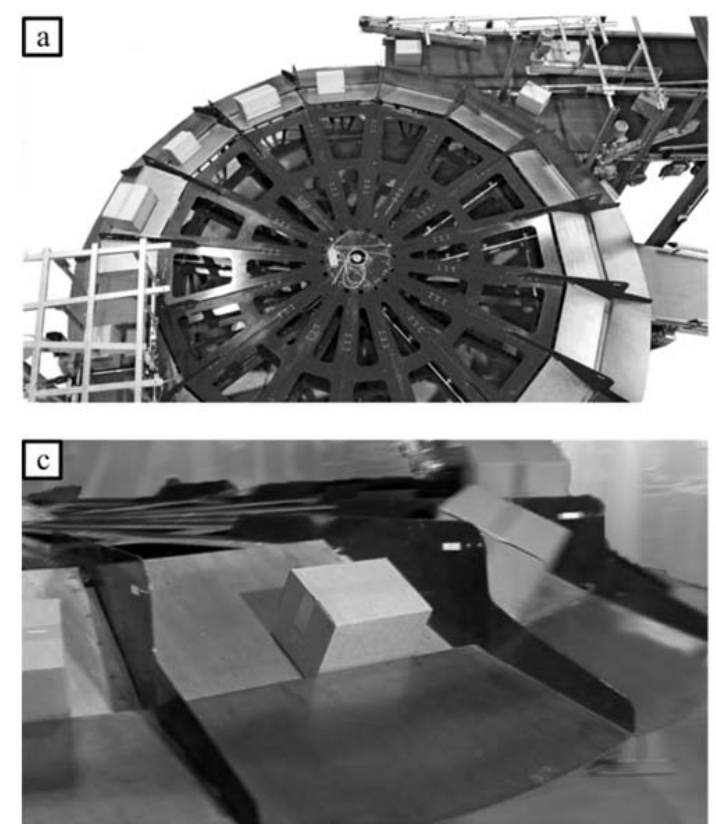

werden über eine Einschleusung, die als horizontale Winkeleinschleusung (Abb.1, Abb.2) oder als Über-KopfEinschleusung (Abb.4a,b) ausgeführt sein kann, auf den Drehsorter übergegeben. Im Bereich der Einschleusung werden die Schwenkklappen durch eine mechanische Führung in eine horizontale Position $\left(0^{\circ}\right)$ zur Gutaufnahme gebracht (Abb.3).
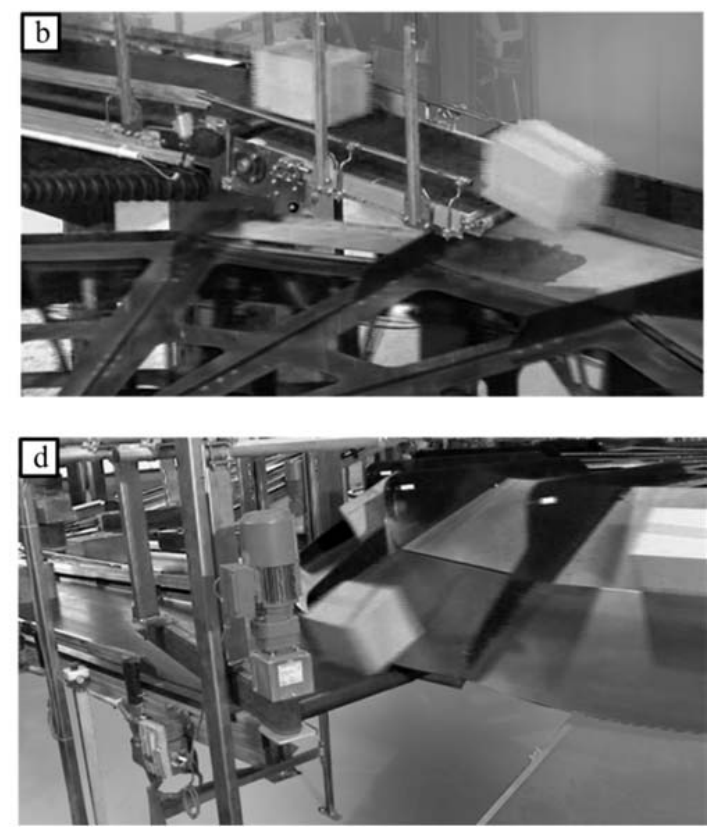

Abbildung 4. Stückgutsortierung mit dem Drehsorter ( $a$-Aufbau; b-Einschleusung; $c$ - Förderung; $d$ - Ausschleusung)

Über die gleiche mechanische Führung wird die Schwenkklappe nach der Übergabe des Gutes auf den
Drehsorter so weit abgesenkt, bis dass ihre Außenkante unterhalb der mitrotierenden Gleitfläche (Neigung $30^{\circ}$ ) 
liegt (Abb.4c). Indem die Schwenkklappe um $5^{\circ}$ pneumatisch angehoben wird (Mikrowurf) bis sie die gleiche Neigung wie die Gleitfläche besitzt, wird das Stückgut ausgeschleust (Abb.4d). Durch Schwerkraft und Zentrifugalkraft beschleunigt (Verhältnis von ca. 7:1 bei Masse $\mathrm{m}=15 \mathrm{~kg}$, Leistung $\mathrm{P}=5000 \mathrm{Stk} . / \mathrm{h}$ ), rutscht das Gut über die mitrotierende Gleitfläche in die Endstelle. Da sich der Drehsorter während der Rutschbewegung des Gutes weiter dreht, ist das Anheben der Schwenkklappe noch vor dem Erreichen der Endstelle erforderlich, um die Drehwinkeländerung zu kompensieren (Offset) [Sch00].

Mit dem beschriebenen Bewegungsablauf des Drehsorters ist es möglich, mit dem Prototypen Stückgüter mit einer Kantenlänge von maximal $600 \mathrm{~mm}$ x $400 \mathrm{~mm}$ x $400 \mathrm{~mm}$ (Länge/Breite/Höhe) und einem Gewicht von maximal $15 \mathrm{~kg} \mathrm{zu}$ sortieren. Der Prototyp erreicht im bestehenden Aufbau eine Winkelgeschwindigkeit $\omega$ von ca. 0,65 1/s bzw. eine Drehzahl n von 375 Umdrehungen/h. Mit den vorhandenen 16 Sortersegmenten $n_{s}$ entspricht dies einer theoretischen Sortierleistung P von $6000 \mathrm{Stk} . / \mathrm{h}$ :

$$
P=\frac{\omega \cdot n_{S}}{2 \pi}=n \cdot n_{S} \rightarrow 375 \frac{1}{h} \cdot 16=6.000 \frac{1}{h}
$$

Bei dieser Leistung werden die Endstellen - verschiedene Rutschen und Förderbänder - zielgenau getroffen. Um diese Sortierleistung tatsächlich zu erreichen, muss die Einschleusung so gestaltet werden, dass sie in der Lage ist, 6000 Teile pro Stunde zielgenau auf den Sorter zu übergeben. Im Folgenden wird vorgestellt, wie diese Einschleusleistung realisiert werden kann.

\section{STAND DER FORSCHUNG}

Nach der Patentanmeldung des Drehsorters im Jahr 1997 erfolgte eine erste einführende Vorstellung des Drehsorterkonzeptes durch Schmidt, in der er von einer theoretischen Sortierleistung von $7000 \mathrm{Stk}$./h bei Stückgutabmessungen von maximal 600mm x 400mm (Länge $\mathrm{x}$ Breite) ausging [Sch97]. Erste Details und spezielle Eigenschaften wie die Einflüsse von Fördergutlänge, Winkelgeschwindigkeit und Segmentanzahl auf Segmentradius und Sorterleistung des Drehsorters wurden 1999 vorgestellt [Sch99]. Hierbei weist Schmidt erstmalig darauf hin, dass die Sorterleistung neben den genannten Parametern auch von Anzahl und Ausführung der Einschleusungen abhängt. In der ersten ausführlichen, theoretischen Betrachtung des Bewegungsverhaltens und der Systemleistung geht Schmidt von einer theoretischen Leistung von 2400 bis $7200 \mathrm{Stk}$./h aus [Sch00]. Bei dieser Überlegung wird eine Einschleusleistung von 2400 Stk./h als maximale Leistung einer getakteten Einschleusung (Stop and Go) angenommen. Durch die Installation mehrerer Einschleusungen ergibt sich eine Vervielfachung der Einschleusleistung, so dass bei drei Einschleusungen eine Gesamteinschleusleistung von 7200 Stk./h realisiert werden könnte. Die Annahme von maximal drei zu installierenden Einschleusungen beruht dabei auf der starken Ver- ringerung der Anzahl der Endstellen, die jede zusätzliche Einschleusung zwangsläufig beim Drehsorterkonzept mit sich bringt. Da die Anzahl der möglichen Endstellen durch den Kreisumfang und die daran anzubringenden Endstellen begrenzt und verglichen mit Sortern in Ringoder Linienstruktur gering ausfällt, wurde die Realisierung und die Leistungserhöhung einer einzelnen Einschleusung angestrebt.

Im Jahr 2002 wurde der Prototyp des Drehsorters fertiggestellt und auf der CeMAT präsentiert. Der Aufbau des Prototyps erfolgte mit einer horizontalen Winkeleinschleusung, mit deren Hilfe die Funktionalität des Drehsorterkonzeptes nachgewiesen werden konnte. Der Prototyp des Drehsorters befindet sich seit 2002 am Versuchsfeld des FLW in Dortmund, wobei die Fa. Lödige Industries im Folgenden die Lizenzrechte erhielt und seitdem den Drehsorter unter dem Namen SpinSorter vertreibt.

Die Verteilleistung des Drehsorters mit einer Einschleusung wird von ten Hompel und Schmidt mit 5000 Stk./h im Jahr 2002 angegeben [HS02a, SW03a, SW03b]. Noch im gleichen Jahr heben sie die Relevanz der Aufbereitung der Stückgutströme und der Synchronisation dieser Ströme mit dem Sorter für die Systemoptimierung hervor [HS02b]. Diese Aussagen unterstreichen die Bedeutsamkeit der Einschleusung in Bezug auf die Ausschöpfung der vollen Leistungsfähigkeit von Sortiersystemen. Folgerichtig wurde im weiteren Verlauf das Bewegungsverhalten der Stückgüter während der Einschleusung zur Bestimmung von Leistungskriterien untersucht. Hierzu gibt Hirschmüller im Jahr 2003 an, dass der Drehsorter bei einer höheren Einschleusleistung mehr als 6000 Stückgüter pro Stunde verteilen könnte. Als Voraussetzung für die Ausschöpfung der maximal möglichen Verteilleistung des Drehsorters, sieht er die Entwicklung einer leistungsfähigeren Einschleustechnik und geeigneter Steuerungsstrategien an [Hir03, SH03]. Die nachfolgenden Untersuchungen am Drehsorter-Prototyp beinhalteten die Optimierung des Bewegungsverhaltens der Stückgüter während der Einschleusung, um die Stückgüter auch bei sehr hohen Geschwindigkeiten zielgenau in die Sortersegmente einzuschleusen [HM04, HJ04]. Hierbei wurden kurzfristig Leistungen bis zu 4000 Stk./h über eine einzelne Einschleusung erzielt. Die Leistungsbeschränkung ergab sich unter anderem durch die verwendete Einschleustechnik. Dabei wurden zwei Varianten untersucht. Die erste Variante bestand in der manuellen Gutaufgabe, mit der Einschleusleistungen von ca. 2400 Stk./h erzielt werden konnten. Die zweite Variante war eine einzelne automatische Einschleusung nach dem Stop and Go Verfahren (getaktete Einschleusung), mit der die oben beschriebene Einschleusleistung von 4000 Stk./h erreicht wurde [HM04]. 


\section{NeUgestaltung DES EINSCHLEUSBEREICHS}

Aus den leistungsbegrenzenden Eigenschaften der bestehenden Einschleustechnik ergab sich die Forderung nach einer automatischen Einschleustechnik, die eine dauerhafte Versorgung des Drehsorters mit mehr als 4000 Teilen pro Stunde sicherstellen kann. Vor diesem Hintergrund wurde der am Lehrstuhl für Förder- und Lagerwesen betriebene Prototyp im Jahr 2009 um ein kreisförmig geschlossenes Stetigfördersystem mit neuartiger Einschleustechnik erweitert (Abb.5). Der hierdurch entstandene Kreislauf befördert die vom Drehsorter ausgeschleusten Güter kontinuierlich bis zur neu gestalteten Einschleusung zurück, von der sie dann wieder direkt auf den Sorter eingeschleust werden [SS10]. Der StetigförderKreislauf bietet somit die Möglichkeit, die Leistung des Drehsorters im Dauerversuch zu untersuchen.

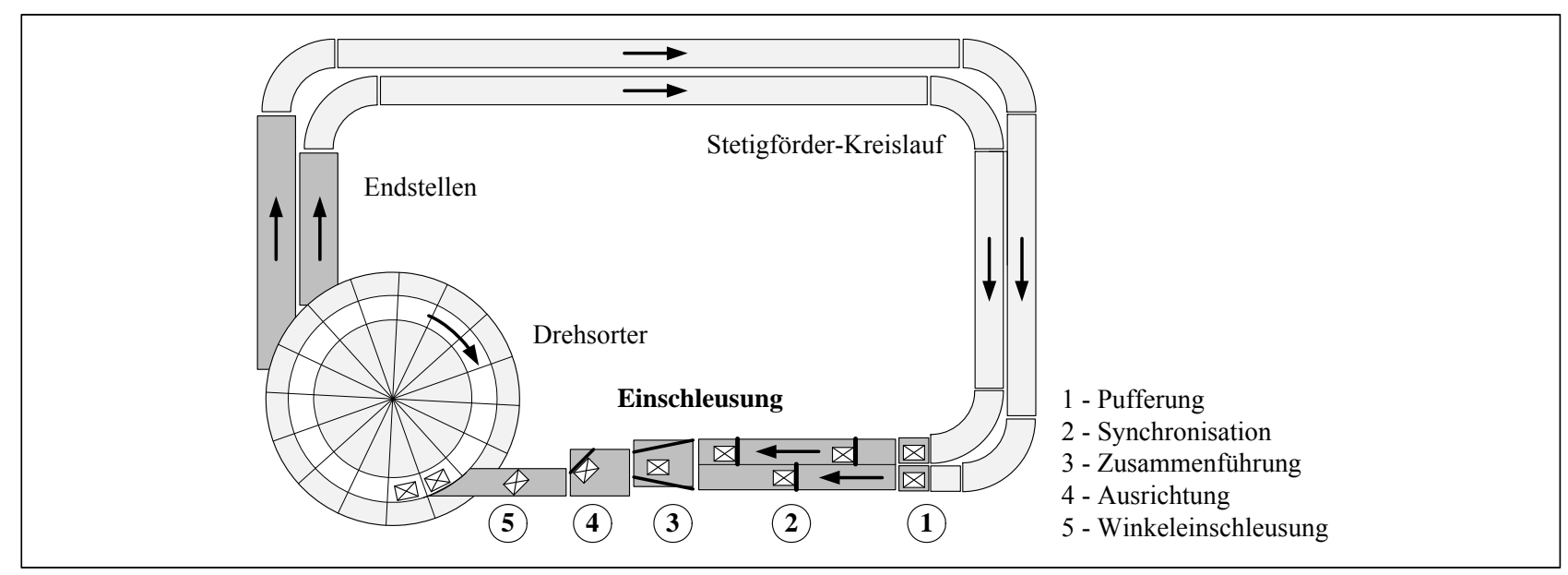

Abbildung 5. Drehsorter mit Stetigförder-Kreislauf und neu gestalteter Einschleusung

Für die Gestaltung der Einschleusung wurden zwei wesentliche Anforderungen formuliert:

- $\quad$ Es muss eine Einschleusleistung von 6000 Stk./h erzielt werden, um die Leistungsfähigkeit des Drehsorters vollständig auszunutzen.

- $\quad$ Ankommende Stückgüter müssen in unregelmäßigen Abständen den Einschleusbereich erreichen, wie es in der Praxis der Fall ist, wenn die Güter von Hand auf die zuführenden Strecken aufgegeben werden.

Um die Anforderungen zu erfüllen, wurde in $\mathrm{Zu}$ sammenarbeit mit den Firmen Lödige Industries, Transnorm und AKDV ein Stetigförder-Kreislauf realisiert, der eine neuartige Einschleusung mit verbesserter Leistung besitzt (Abb.5).

Die zu verteilenden Güter können vom im Uhrzeigersinn rotierenden Drehsorter auf die Endstellen, zwei horizontal verlaufende Bandförderer, verteilt werden, um dem Stetigförder-Kreislauf zugeführt zu werden. Von den horizontalen Bandförderern werden die Güter über Stetigförderer in zwei parallel verlaufenden Strecken bis zur Einschleusung gefördert. Der entstandene StetigförderKreislauf ermöglicht die Rückführung der Güter und die Untersuchung der Einschleusleistung in den DrehsorterPrototypen im Dauerversuch.

Die Einschleusung beginnt mit der Pufferung der Güter auf zwei kurzen Förderbändern, von denen aus die
Stückgüter zur Synchronisation mit dem Drehsorter auf einen Taktförderer (Positioner) übergeben werden (Abb.52). Nach der Zusammenführung der zwei Stückgutströme (Abb.5-3) werden die Güter an einem senkrecht über dem Förderband stehenden angetriebenen Bandabweiser im Winkel von $45^{\circ}$ ausgerichtet, um in diesem Winkel auf den Drehsorter gefördert zu werden (Abb.5-4). Die 45Orientierung der Stückgüter ergibt sich aus dem Winkel des Dreiecksbandes. Im Folgenden werden diese drei für eine gutschonende und präzise Einschleusung maßgeblichen Prozesse näher beleuchtet.

\subsection{SYNCHRONISATION}

Um Stückgüter präzise auf die Schwenkklappen einzuschleusen, muss die Stellung des Drehsorters zum Zeitpunkt der Einschleusung des Gutes einer genau definierten Position entsprechen. Dies wird zum einen durch ein fest vorgegebenes Verhältnis der Geschwindigkeiten von Drehsorter und Einschleusung erreicht. Zum anderen muss das Stückgut genau zu dem Zeitpunkt an der Vorderkante des Dreiecksbandes eintreffen, an dem das Sortersegment eine fest vorgegebene Position zum Dreiecksband erreicht hat, damit eine dynamische Übergabe des Stückguts erfolgen kann.

Um dies zu realisieren, wird der Taktförderer (Positioner) der Fa. Transnorm eingesetzt (Abb.6). 


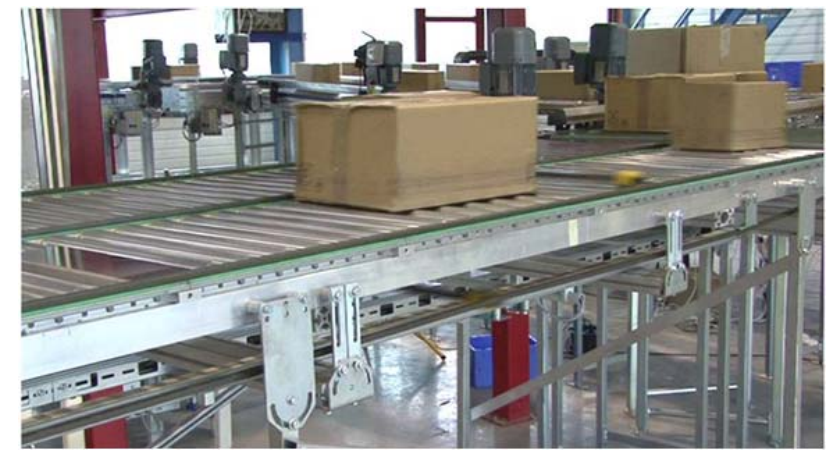

Abbildung 6. Taktförderer (Positioner), Fa. Transnorm

Der Positioner besteht aus zwei parallel angeordneten nicht angetriebenen Rollenbahnen und drei in Förderrichtung vertikal umlaufenden, angetriebenen Ketten, zwischen denen Vierkantprofile (Mitnehmer) angebracht sind. Die Mitnehmer sind versetzt in immer gleichen $\mathrm{Ab}$ ständen auf beiden Förderstrecken angebracht und bewegen sich mit den Ketten um die Rollenbahn herum. Werden Stückgüter von den Pufferbändern auf den Positioner übergeben, rollt das Gut zunächst aus, bis es von einem umlaufenden Mitnehmer von hinten erfasst und formschlüssig bis zum Ende des Positioners geführt wird. Dadurch werden die Stückgüter in einen durch die Mitnehmer fest definierten, immer gleich großen Abstand (Hinterkante zu Hinterkante) gebracht.

Da die Mitnehmer auf den zwei parallelen Förderstrecken versetzt angeordnet sind, kann im Anschluss an den Positioner mit einem Zusammenführungselement die Aggregation der Güterströme im Reißverschlussverfahren erfolgen. Der so erzeugte Paketstrom hat eine konstante Geschwindigkeit und einen fest vorgegeben Abstand zwischen den Hinterkanten aller Pakete. Aufgrund der formschlüssigen Verbindung zwischen Mitnehmer und Paket führt eine Synchronisation der Mitnehmer mit den Positionen der Sortersegmente zu dem oben beschriebenen Effekt.

Zur Synchronisation von Drehsorter und Mitnehmer werden zunächst die Positionen der Sortersegmente und die Geschwindigkeit am Drehsorter gemessen. Sobald diese Daten in der Drehsortersteuerung vorliegen, wird mittels TCP/IP ein Sync-Impuls an die Steuerung des Positioners übermittelt. Daraufhin beschleunigen der Positioner bzw. seine Ketten mit den Mitnehmern auf die zur Drehsorterleistung passende Geschwindigkeit. Die Positionen der Sortersegmente wie auch die Positionen der Mitnehmer werden fortlaufend über Inkrementalgeber exakt bestimmt. Die Geschwindigkeit des Positioners wird nun so lange ausgeregelt (beschleunigt), bis die Positionen von Mitnehmern und Sortersegmenten synchronisiert sind. Im laufenden Betrieb wird die Synchronisation über einen Regelkreis sichergestellt. Die beschriebene Prozedur ist somit nur ein einziges Mal nach dem Start der Anlage bzw. nach der Einstellung einer neuen Geschwindigkeitsstufe notwendig.

\subsection{AUSRICHTUNG}

Um eine optimale Platzausnutzung in dem Schalensegment zu erreichen, müssen die Packstücke vor dem Einschleusvorgang ausgerichtet werden. Die Ausrichtung erfolgt mittels eines senkrecht über einem Bandförderer angebrachten motorischen Bandabweisers, der in einem Winkel von ca. $45^{\circ}$ zur Förderrichtung angebracht ist (Abb.7). Beim Abweisvorgang bewegt sich das Stückgut zunächst mit der konstanten Bandgeschwindigkeit auf den Abweiser zu, bis die vordere Kante des Stückgutes auf den Abweiser trifft. Nach dem ersten Zusammenstoß vollzieht das Stückgut eine zusammengesetzte Bewegung aus Rotation und Translation oder eine reine Rotation. Durch die Rotation dreht sich das Stückgut, bis es mit einer Seite am Abweiser anliegt. Je nach Geschwindigkeit und Umlaufrichtung des am Abweiser umlaufenden Bandes vollführt das Stückgut im Folgenden eine translatorische Bewegung. Während all dieser Bewegungsphasen werden die Bewegungsgrößen des Stückgutes verändert [Mül80, Möh83].

Da im beschriebenen Aufbau keine signifikanten Unterschiede zwischen verschiedenen Stückgütern in ihrem Bewegungsverhalten am Bandabweiser bestehen, kann die Synchronisation zwischen Positioner und Drehsorter unabhängig von den verwendeten Stückgütern erfolgen.

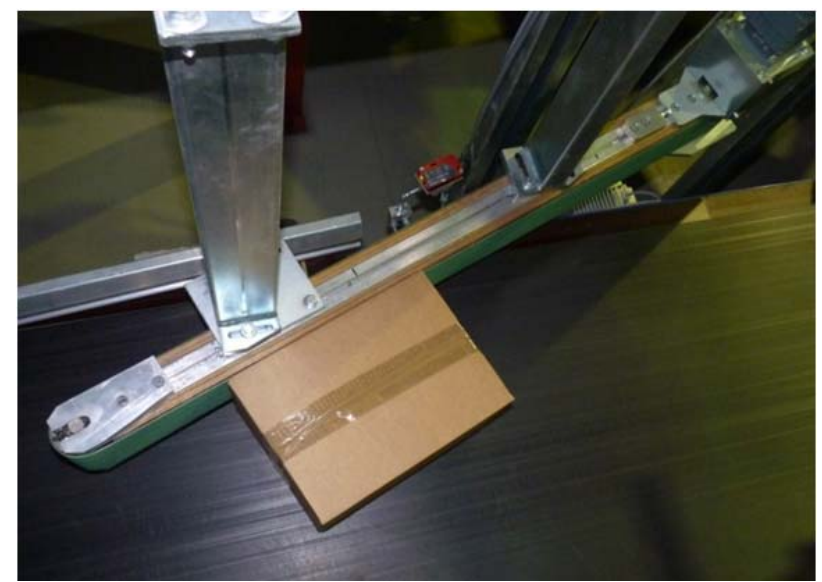

Abbildung 7. Ausrichtung mittels Bandabweiser (Draufsicht)

\subsection{WINKELEINSCHLEUSUNG}

Durch eine horizontale Übergabe vom Einschleusband auf den Drehsorter wird eine gutschonende Einschleusung gewährleistet. Für die Übergabe wurde ein Winkel zwischen Einschleusband und Drehsorter von $45^{\circ}$ gewählt (Abb.8). Bei größeren Einschleuswinkeln ergeben sich zu hohe Zuführ- und Radialgeschwindigkeiten, um die Tangentialgeschwindigkeit aufbringen zu können. Im Gegensatz dazu wird bei kleineren Einschleuswinkeln die Radialgeschwindigkeit zu klein, so dass das Gut nicht weit genug auf die Schwenkklappe gleitet. Die Übergabe auf den Drehsorter wird mit einem Dreiecksband mit Rundriemen realisiert. 
Werden die Stückgüter mit einer $45^{\circ}$-Orientierung in das Sortersegment übergeben, kommen sie mit ihrer Längsseite parallel zur Kante der Schwenkklappe zum
Liegen. Dies ist für die eine präzise Ausschleusung eine wichtige Voraussetzung [Hir03].

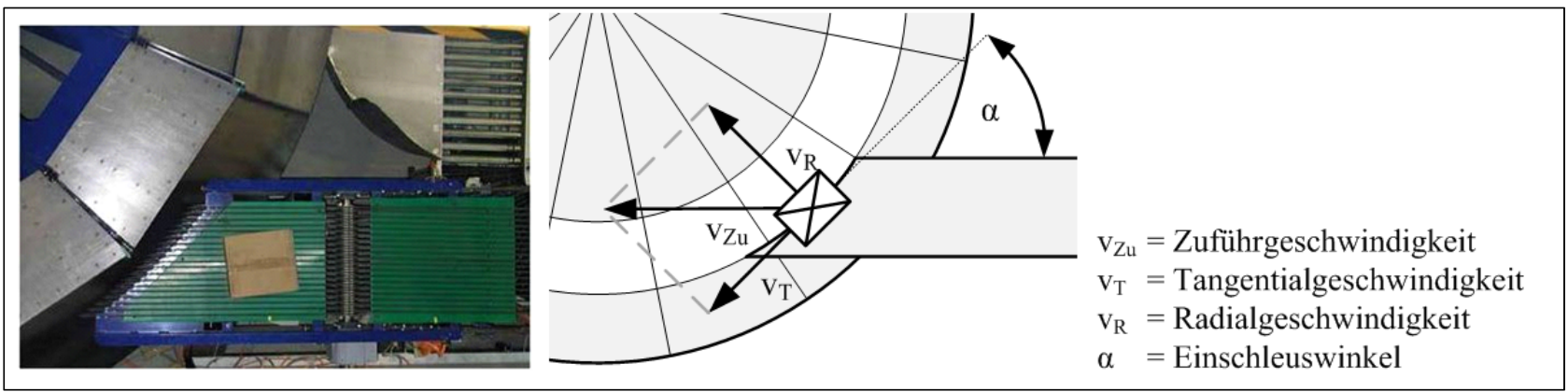

Abbildung 8. Winkeleinschleusung mit Einschleusgeschwindigkeiten, nach [Hir03]

$\mathrm{Zu}$ beachten ist bei der horizontalen Winkeleinschleusung, dass die Geschwindigkeit des Einschleusbandes $\mathrm{v}_{\mathrm{Zu}}$ so groß gewählt wird, dass die tangentiale Geschwindigkeitskomponente des Stückguts $\mathrm{v}_{\mathrm{T}}$ der Tangentialgeschwindigkeit des Drehsorters entspricht. Der Zusammenhang der Geschwindigkeiten bei der Einschleusung unter dem Winkel $\alpha$ nach Abbildung 6 ergibt sich zu:

$$
v_{Z u}=\frac{v_{T}}{\cos (\alpha)}
$$

Der Drehsorter-Prototyp dreht mit einer Winkelgeschwindigkeit $\omega$ von $0,651 / \mathrm{s}$ und besitzt einen Radius $\mathrm{r}_{\mathrm{T}}$ von 2,6 $\mathrm{m}$ an den Schalenaußenkanten, woraus eine Tangentialgeschwindigkeit $\mathrm{v}_{\mathrm{T}}$ von $1,7 \mathrm{~m} / \mathrm{s}$ bei einer Leistung $\mathrm{P}$ von 6000 Stk./h resultiert.

$$
v_{T}=\omega \cdot r_{T} \rightarrow 0,65 \frac{1}{s} \cdot 2,6 m=1,7 \frac{m}{s}
$$

Das bedeutet, dass die Zuführgeschwindigkeit $\mathrm{v}_{\mathrm{Zu}}$ bei der $45^{\circ}$-Winkeleinschleusung mindestens

$$
v_{Z u}=\frac{v_{T}}{\cos (\alpha)} \rightarrow \frac{1,7 \frac{m}{s}}{\cos \left(45^{\circ}\right)}=2,4 \frac{m}{s}
$$

betragen muss, um die Stückgüter im $45^{\circ}$-Winkel auf den Drehsorter einzuschleusen. Hierbei ist jedoch zu berücksichtigen, dass sich die Geschwindigkeitsverhältnisse des Stückgutes während des Übergangs zum Drehsorter stetig ändern. Die Tangential- und Radialgeschwindigkeit reduzieren sich aufgrund der Gleitreibung genauso wie die Geschwindigkeitskomponente des Drehsorters infolge des abnehmenden Radius immer weiter [Hir03].

Ein weiterer für die Bestimmung der Zuführgeschwindigkeit $\mathrm{v}_{\mathrm{Zu}}$ wichtiger Einflussfaktor ist die durch unterschiedliche Stückgutbreiten bedingte Mittenabweichung in $90^{\circ}$ zur Förderrichtung der Einschleusung.

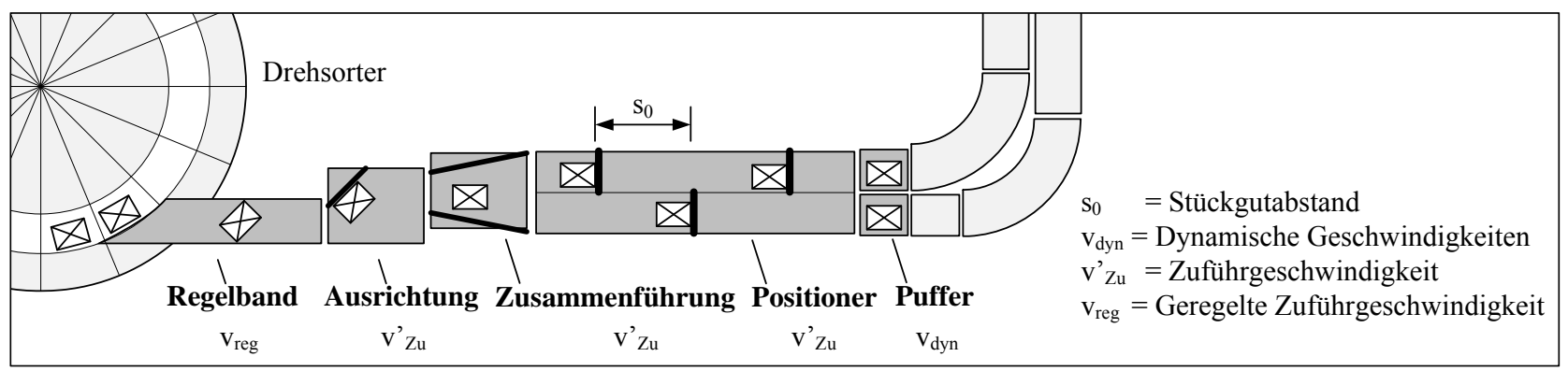

Abbildung 9. Prinzipdarstellung Einschleusbereich mit Winkeleinschleusung

Auf der Grundlage der theoretischen und experimentellen Ergebnisse von Hirschmüller [Hir03] und weiteren experimentellen Untersuchungen wurde der Wert für die Zuführgeschwindigkeit $\mathrm{v}^{{ }^{\prime} \mathrm{zu}}$ auf

$$
v_{Z u}^{\prime}=2,6 \frac{\mathrm{m}}{\mathrm{s}}
$$

festgelegt. Für den Einschleusbereich werden damit die Zuführgeschwindigkeit $\mathrm{v}^{\star} \mathrm{Zu}$ und die Durchsatzleistung $\mathrm{P}$ vorgegeben, so dass der konstante Stückgutabstand der
Stückgüter $\mathrm{s}_{0}$ im Einschleusbereich berechnet werden kann.

$$
s_{0}=\frac{v_{Z u}}{P} \rightarrow \frac{2,6 \frac{m}{s}}{6.000 \frac{1}{h}}=1,6 \mathrm{~m}
$$

Der Abstand von 1,6 m entspricht dem Abstand zwischen zwei versetzt auf dem Positioner angebrachten Mitnehmern, durch die der Abstand der Hinterkanten der Stückgüter bestimmt wird (Abb.9). 


\subsection{VERSUCHSERGEBNISSE BEI WINKELEINSCHLEUSUNG}

Mit der Kombination aus Pufferbändern, Positioner, Zusammenführung, Ausrichtung und Regelband wurde eine neuartige Einschleustechnik konzipiert und realisiert, die es ermöglicht, quaderförmige Stückgüter von bis zu $600 \mathrm{~mm}$ Länge im Dauerbetrieb auf den Drehsorter fehlerfrei zuzuführen. Hierzu wurden alle Förderer im Einschleusbereich mit der notwendigen Antriebstechnik ausgestattet, um die für die Leistung von 6000 Stk./h erforderliche Geschwindigkeit von bis zu 2,6 m/s zu erzielen.

Im Leistungsbereich bis $4000 \mathrm{Stk} . / \mathrm{h}$ werden die Stückgüter exakt in die Schalensegmente des Drehsorters übergeben. Hierzu werden die Güter durch den Positioner formschlüssig mit dem Drehsorter synchronisiert und weiter befördert, ohne dass durch Zusammenführung oder Ausrichtung die Synchronisation wesentlich beeinflusst würde. Die Güter werden ununterbrochen an den Positioner übergeben, wodurch alle Lücken zwischen den Mitnehmern des Positioners besetzt und somit auch alle Sortersegmente belegt werden. Hierdurch werden die Leistung der Einschleusung und somit auch die Leistung des Drehsorters von 4000 Stk./h voll ausgeschöpft.

Für eingestellte Leistungen von 5000 Stk./h und 6000 Stk./h werden die Stückgüter zwar weiterhin ohne Probleme von den Pufferbändern auf den Positioner übergeben und von diesem synchron zum Drehsorter befördert, allerdings werden die Stückgüter am Bandabweiser nicht mehr ausreichend ausgerichtet. Die mangelnde Ausrichtung liegt zum einen in der hohen Zugkraft in Förderrichtung, verursacht durch die hohe Geschwindigkeit des Förderbandes, wodurch das Stückgut nach der Berührung mit dem Abweiser wieder in Längsrichtung gezogen wird. Zum anderen stoßen die Stückgüter mit hohen Geschwindigkeiten gegen den Abweiser, was eine translatorische Ablenkung in $90^{\circ}$ zur Förderrichtung verursacht. Diese beiden Effekte verhindern eine sichere und genaue Einschleusung für das angestrebte Spektrum quaderförmiger Stückgüter.

Um die Leistung von 6000 Stk./h im Einschleusbereich zu realisieren, sind zwei Möglichkeiten für eine Veränderung der Einschleusung gegeben. Zum einen kann versucht werden, die Ausrichtungstechnik zu verbessern, um eine Orientierung der Stückgüter von $45^{\circ}$ bei Ge- schwindigkeiten des Förderbandes von 2,6 m/s zu erreichen. Zum anderen besteht die Möglichkeit, die Stückgüter tangential von oben in den Drehsorter einzuschleusen (Über-Kopf-Einschleusung).

\section{VARIATION DER EINSCHLEUSTECHNIK}

Die in Kapitel 3 vorgestellte Einschleustechnik genügte aufgrund der mangelnden Ausrichtung der Stückgüter bei hohen Geschwindigkeiten nicht den Leistungsanforderungen. Aus diesem Grund wurde eine Über-KopfEinschleusung realisiert, bei der die Stückgüter nicht in $45^{\circ}$ ausgerichtet werden müssen, da sie längsausgerichtet tangential von oben auf den Drehsorter übergeben werden (Abb.10).
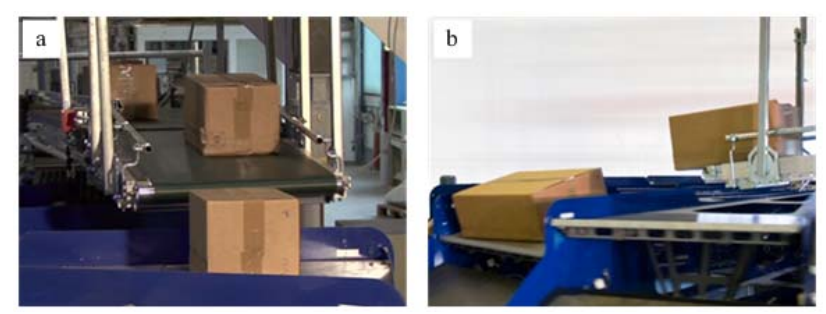

Abbildung 10. Über-Kopf-Einschleusung, a) Ansicht von vorne b) Seitenansicht

\section{1 ÜBER-KOPF-EINSCHLEUSUNG}

Die Über-Kopf-Einschleusung ermöglicht eine Übergabe der Pakete in tangentialer Bewegungsrichtung des Drehsorters, wodurch die $45^{\circ}$-Ausrichtung der Pakete entfällt und die Pakete längsausgerichtet eingeschleust werden (Abb.11). Durch die Über-Kopf-Einschleusung ändert sich der mechanische Aufbau der Fördertechnik insofern, als dass die Förderer der Einschleusung tangential auf den Drehsorter zulaufen und eine Höhenanpassung der Fördertechnik durch die Einschleusung von oben notwendig wird. Außerdem sind die Überlegungen zur Zerlegung der Geschwindigkeitskomponenten, wie sie in Kapitel 3.3 angestellt wurden, nicht mehr nötig. Die Zuführgeschwindigkeit der Stückgüter $\mathrm{v}_{\mathrm{Zu}}{ }^{*}$ entspricht bei der Über-KopfEinschleusung genau der Tangentialgeschwindigkeit des Drehsorters in der Mitte der Schalensegmente $\mathrm{v}_{\mathrm{T}}{ }^{*}$ (Abb.10). Die Geschwindigkeit $\mathrm{v}_{\mathrm{T}} *$ ist damit $\mathrm{zu}$ unterscheiden von der Tangentialgeschwindigkeit des Drehsorters am Rand der Schalensegmente $\mathrm{v}_{\mathrm{T}}$ wie sie in Kapitel 3.3 beschrieben wird.

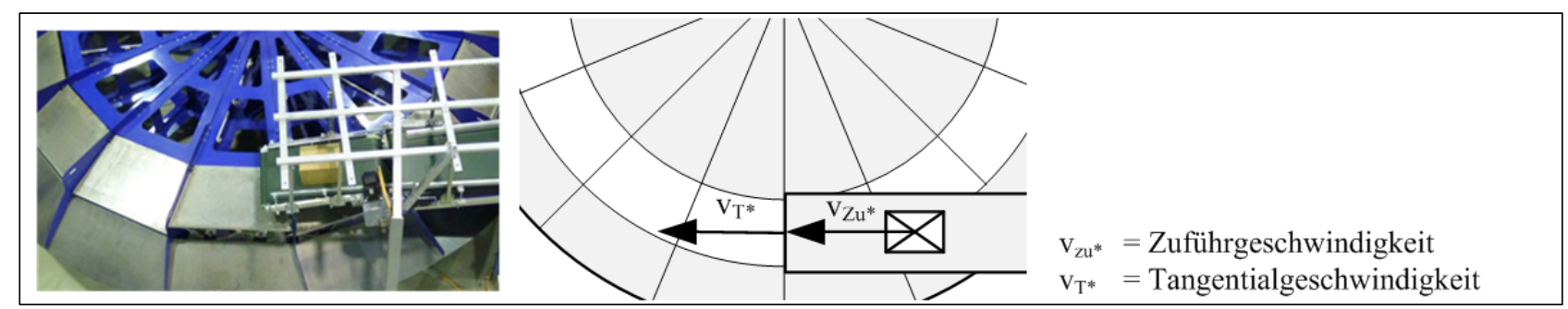

Abbildung 11. Über-Kopf-Einschleusung mit Einschleusgeschwindigkeiten 
Für Gleichung 3.1 gilt im Fall der Über-KopfEinschleusung mit einem Einschleuswinkel von $\alpha=0^{\circ}$ :

$$
v_{Z u^{*}}=\frac{v_{T^{*}}}{\cos (\alpha)} \rightarrow \frac{v_{T^{*}}}{\cos (0)}=v_{T^{*}}
$$

Für die Leistung von 6000 Stk./h ergibt sich eine Tangentialgeschwindigkeit $\mathrm{v}_{\mathrm{T}} *$ der Schalenmitten des Drehsorters von $1,5 \mathrm{~m} / \mathrm{s}$ bei einem Radius $\mathrm{r}_{\mathrm{T}}{ }^{*}$ von $2,3 \mathrm{~m}$.

$$
v_{T^{*}}=\omega \cdot r_{T^{*}} \rightarrow 0,65 \frac{1}{\mathrm{~s}} \cdot 2,3 \mathrm{~m}=1,5 \frac{\mathrm{m}}{\mathrm{s}}
$$

Da nach Gleichung 4.1 die Tangentialgeschwindigkeit $\mathrm{v}_{\mathrm{T}}{ }^{*}$ der Zuführgeschwindigkeit $\mathrm{v}_{\mathrm{Zu}}{ }^{*}$ bei der Über-KopfEinschleusung entspricht, gilt

$$
v_{Z u^{*}}=1,5 \frac{\mathrm{m}}{\mathrm{s}}
$$

Da die Geschwindigkeiten des Drehsorters (Schalenmitte) und der Stückgüter auf dem letzten Einschleusband gleich groß sein müssen, um die Schwenkklappen des Drehsorters exakt zu belegen, muss auch der Abstand der Güter auf beiden Förderern gleich groß sein. Dieser Abstand ist genau die Länge eines Kreisbogensegmentes $\mathrm{s}_{1}$ im Radius $\mathrm{r}_{\mathrm{T}}{ }^{*}$. Mit der Zuführgeschwindigkeit $\mathrm{v}_{\mathrm{Zu}}{ }^{*}$ und dem Abstand $\mathrm{s}_{1}$ lässt sich die Leistung $\mathrm{P}$ bestimmen:

$$
P=\frac{v_{Z u^{*}}}{s_{1}} \rightarrow \frac{1,5 \frac{m}{s}}{0,9 m}=6.000 \frac{1}{h}
$$

Der Abstand der Stückgüter wird somit genau auf den Abstand der Sortersegmente auf dem Kreisbogen des Drehsorters bei einem Radius $\mathrm{r}_{\mathrm{T}}{ }^{*}$ von 2,3 m angepasst. Dass bedeutet, dass das letzte Einschleusband die einzuschleusenden Stückgüter genau mit der Geschwindigkeit und dem Abstand befördert, wie sie auch auf dem Drehsorter vorliegen. Dazu muss das letzte Band langsamer als die vorhergehenden Bänder laufen, damit der Abstand der Stückgüter vor dem Drehsorter von der bestehenden Teilung des Positioners von 1,6 m $\left(\mathrm{s}_{0}\right)$ auf $0,9 \mathrm{~m}$ $\left(\mathrm{s}_{1}\right)$ reduziert wird (Abb.12).

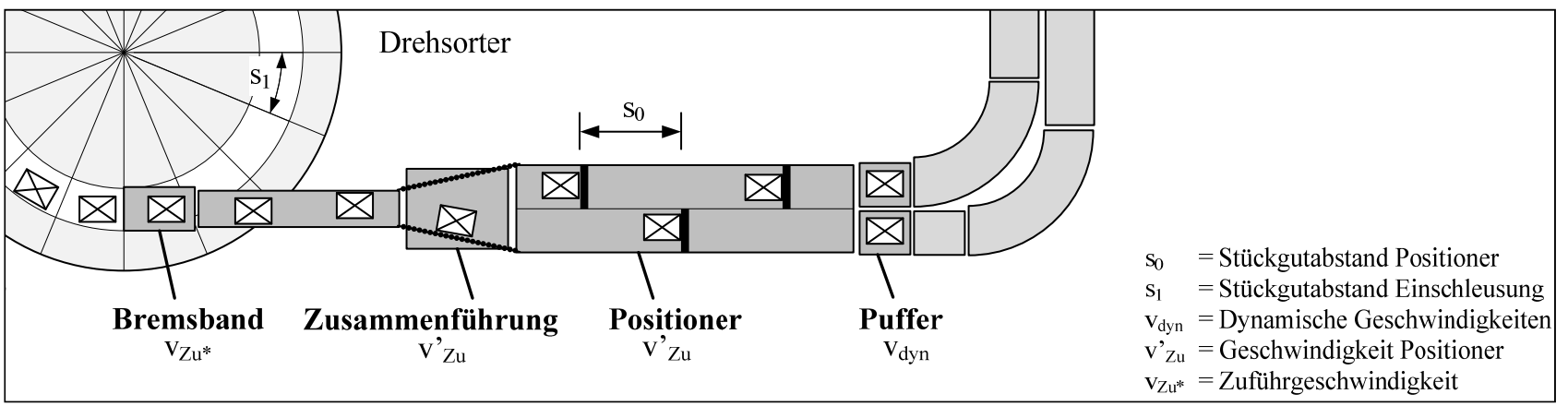

Abbildung 12. Prinzipdarstellung Einschleusbereich mit Über-Kopf-Einschleusung

\subsection{VERSUCHSERGEBNISSE BEI ÜBER-KOPF- EINSCHLEUSUNG}

Die Veränderung der Einschleustechnik von Winkeleinschleusung zu Über-Kopf-Einschleusung bringt eine Veränderung der Leistungsparameter mit sich. Der durch den Positioner geschaffene Abstand von 1,6 m bei einer Geschwindigkeit von $2,6 \mathrm{~m} / \mathrm{s}$ wird bei der Über-KopfEinschleusung auf $0,9 \mathrm{~m}$ bei $1,5 \mathrm{~m} / \mathrm{s}$ reduziert. Eine Ausrichtung der Stückgüter im $45^{\circ}$-Winkel ist nicht mehr nötig, da die Stückgüter längsausgerichtet eingeschleust werden.

Der Aufbau mit Über-Kopf-Einschleusung zeigt, dass die Stückgüter trotz des starken Abbremsvorgangs auf dem Bremsband exakt in die Mitte der Schwenkklappen eingeschleust werden. Die exakte Einschleusung trifft auch auf die hohen Leistungsstufen $\mathrm{zu}$.

Im Versuchsaufbau mit Über-Kopf-Einschleusung konnte in einer einstündigen Messung eine maximale Leistung von 5681 Stk./h erzielt werden. Hierzu wurden als Versuchsgüter quaderförmige Kartons aus Wellpappe mit den Abmessungen 310 mm x 220 mm x 200 mm und einem Gewicht von ca. $3 \mathrm{~kg}$ verwendet. Im Kurzzeitbereich zeigte sich, dass für etwa 15 Kartons hintereinander jede Schale getroffen werden konnte, bis die erste Schale nicht mehr belegt werden konnte. Das Aussetzen der Gutzufuhr liegt in dem Aufbau der Versuchsanlage als Kreislauf begründet, wodurch nicht ausreichend Kartons zum Drehsorter beständig über einen längeren Zeitraum gefördert werden konnten. Hier kam es immer wieder zu Steuerungstechnischen Problemen, die zu einem kurzen Abriss des Materialstroms führten. 


\section{ZUSAMMENFASSUNG UND AUSBLICK}

Das Ziel der Neugestaltung des Einschleusbereichs des Drehsorter-Prototypen am Lehrstuhl für Förder- und Lagerwesen war die Realisierung einer neuartigen Einschleustechnik, die es ermöglicht, 6000 Teile pro Stunde einzuschleusen und damit die volle Leistungsfähigkeit des Drehsorters mit einer Einschleusung auszuschöpfen. Die Über-Kopf-Einschleusung und der Positioner bilden die wesentlichen Komponenten des neuen Einschleusbereichs, wodurch die Stückgüter mit einfacher Technik synchron gefördert und exakt in die Schwenkklappen des Drehsorters bei maximaler Leistung eingeschleust werden.

Neben der Über-Kopf-Einschleusung steht weiterhin die Winkeleinschleusung zur Verfügung, die eine vorhergehende Ausrichtung der Stückgüter erfordert. Mit der Winkeleinschleusung kann eine Sortierleistung von 4000 Stk./h erreicht werden. Bei höheren Leistungen wirkt sich die Technik zum Ausrichten der Stückgüter leistungsbeschränkend aus, weshalb die Winkeleinschleusung für Leistungen über $4000 \mathrm{Stk}$./h bisher nicht geeignet ist.

Beim Vergleich der Über-Kopf-Einschleusung mit der Winkeleinschleusung sind für beide Einschleusvarianten die Einsatzbereiche und -merkmale gegenüberzustellen und für den Einsatzfall abzuwägen. Insbesondere stehen der gutschonenden Übergabe und Rezirkulationsmöglichkeit mit Winkeleinschleusung bei der Über-KopfEinschleusung die Einschleusung ohne Ausrichtung und eine höhere Leistung gegenüber.

Mit dem neugestalteten Einschleusbereich wurde der Nachweis für die Leistungsfähigkeit des Drehsorters von 6000 Stk./h mit nur einer Einschleusung erbracht. Die Ausweitung des Leistungsbereichs eröffnet zukünftig eine Ausweitung des Einsatzbereichs des Drehsorters. Derzeit werden weitere leistungssteigernde Einflüsse auf Sortiersysteme am Lehrstuhl für Förder- und Lagerwesen untersucht.
FORMELZEICHEN

Formelzeichen Einheit Name

\begin{tabular}{|c|c|c|}
\hline B & {$[\mathrm{m}]$} & Gutbreite \\
\hline $\mathrm{L}$ & {$[\mathrm{m}]$} & Gutlänge \\
\hline $\mathrm{P}$ & {$[1 / \mathrm{h}]$} & Sortierleistung, Durchsatz \\
\hline $\mathrm{n}$ & {$[1 / \mathrm{h}]$} & $\begin{array}{l}\text { Drehzahl } \\
\text { (Umdrehungen pro Stunde) }\end{array}$ \\
\hline $\mathrm{n}_{\mathrm{S}}$ & {$[-]$} & $\begin{array}{l}\text { Anzahl Plätze auf dem } \\
\text { Drehsorter }\end{array}$ \\
\hline $\mathrm{r}_{\mathrm{T}}$ & {$[\mathrm{m}]$} & Radius (Schalenaußenkante) \\
\hline $\mathrm{r}_{\mathrm{T}^{*}}$ & {$[\mathrm{~m}]$} & Radius (Schalenmitte) \\
\hline $\mathrm{s}_{0}$ & {$[\mathrm{~m}]$} & Stückgutabstand (Positioner) \\
\hline $\mathrm{s}_{1}$ & {$[\mathrm{~m}]$} & $\begin{array}{l}\text { Stückgutabstand } \\
\text { (Über-Kopf-Einschleusung) }\end{array}$ \\
\hline $\mathrm{V}_{\mathrm{dyn}}$ & {$[\mathrm{m} / \mathrm{s}]$} & $\begin{array}{l}\text { Dynamische Geschwindig- } \\
\text { keiten }\end{array}$ \\
\hline $\mathrm{V}_{\mathrm{R}}$ & {$[\mathrm{m} / \mathrm{s}]$} & Radialgeschwindigkeit \\
\hline $\mathrm{V}_{\text {reg }}$ & {$[\mathrm{m} / \mathrm{s}]$} & $\begin{array}{l}\text { Geregelte Zuführgeschwin- } \\
\text { digkeit }\end{array}$ \\
\hline $\mathrm{v}_{\mathrm{T}}$ & {$[\mathrm{m} / \mathrm{s}]$} & $\begin{array}{l}\text { Tangentialgeschwindigkeit } \\
\text { (Schalenaußenkante) }\end{array}$ \\
\hline $\mathrm{V}_{\mathrm{T}^{*}}$ & {$[\mathrm{~m} / \mathrm{s}]$} & $\begin{array}{l}\text { Tangentialgeschwindigkeit } \\
\text { (Schalenmitte) }\end{array}$ \\
\hline $\mathrm{V}_{\mathrm{Zu}}$ & {$[\mathrm{m} / \mathrm{s}]$} & $\begin{array}{l}\text { Zuführgeschwindigkeit } \\
\text { (Winkeleinschleusung, } \\
\text { theoretisch) }\end{array}$ \\
\hline $\mathrm{V}_{\mathrm{Zu}^{*}}$ & {$[\mathrm{~m} / \mathrm{s}]$} & $\begin{array}{l}\text { Zuführgeschwindigkeit } \\
\text { (Über-Kopf-Einschleusung) }\end{array}$ \\
\hline$v^{6} \mathrm{Zu}$ & {$[\mathrm{m} / \mathrm{s}]$} & $\begin{array}{l}\text { Zuführgeschwindigkeit } \\
\text { (Winkeleinschleusung, } \\
\text { experimentell) }\end{array}$ \\
\hline$\alpha$ & {$\left[{ }^{\circ}\right]$} & Einschleuswinkel \\
\hline$\delta$ & {$\left[{ }^{\circ}\right]$} & $\begin{array}{l}\text { Öffnungswinkel eines Scha- } \\
\text { lensegments am Drehsorter }\end{array}$ \\
\hline$\omega$ & {$[1 / \mathrm{s}]$} & Winkelgeschwindigkeit \\
\hline
\end{tabular}




\section{LITERATUR}

[Hir03]

[HJ04]

[HM04]

[HS02a]

[HS02b]

[JH06]

[Möh83]

[Mül80]

[Sch97]

[Sch99]

[Sch00]

[SH03]

[SS10]

[SW03a]

[SW03b]

[VDI3619]
Hirschmüller, Thomas: Leistungskriterien und Systemfindung für die Stückguteinschleusung auf Sorter. Dissertation, Technische Universität Dortmund, 2003.

ten Hompel, Michael; Jodin, Dirk: Leistungsreserven stecken im Detail - Kinematik der Sortiersysteme 2. Berlin: Huss-Medien GmbH. In: Hebezeuge und Fördermittel, Jahrgang 44, 11/ 2004, S.608-611.

ten Hompel, Michael; Mayer, Andreas: Strategy optimization at sorting system feeding station. In: Graduate School of Production Engineering and Logistics, Research Report. Technische Universität Dortmund. 2004, S.18-21.

ten Hompel, Michael; Schmidt, Thorsten: Das Drehsorterkonzept - Alternative zum Verteilen von Kleingut. Berlin: Huss-Medien GmbH. In: Hebezeuge und Fördermittel, Jahrgang 42, 2002, S.150-152.

ten Hompel, Michael; Schmidt, Thorsten: Sortier- und Verteilsysteme in Wissenschaft und Forschung. In: Fördern und Heben, Jahrgang 52, 9/ 2002, S.571-573.

Jodin, Dirk; ten Hompel, Michael: Sortier- und Verteilsysteme. Berlin Heidelberg: Springer-Verlag 2006.

Möhle, Bernd: Zum Bewegungsverhalten von Stückgütern auf Gurtförderern beim Ausrichten und Abweisen. Dissertation, Technische Universität Carolo-Wilhelmina zu Braunschweig, 1983.

Müller, Heinz: Untersuchungen zum Bewegungsverhalten von Einzellasten auf Gurtförderern beim Abweisvorgang. Dissertation, Technische Universität CaroloWilhelmina zu Braunschweig, 1980.

Schmidt, Thorsten: Rotieren und Sortieren. Fördertechnik '97, Sonderpublikation des Fördermittel Journals, Frankfurt/Main, 1997, S.36-37.

Schmidt, Thorsten: Rotierend verteilen. Berlin: Huss-Medien GmbH. In: Hebezeuge und Fördermittel, Jahrgang 39, 3/ 1999, S.74-75.

Schmidt, Thorsten: Stückgutverteilung nach dem Drehsorterkonzept - Bewegungsverhalten und Systemleistung. Dissertation, Technische Universität Dortmund, 2000.

Schmidt, Thorsten; Hirschmüller, Thomas: Konzeption kostengünstiger Verteiltechniken am Beispiel des Drehsorters. In: Jahrbuch der Logistik, 2003, S. 237-241.

Semrau, Kai F.; Sadowsky, Volker: Drehsorter neu aufgelegt - bis zu 6.000 Pakete/h eingeschleust und sortiert. In: Tagungsband auf CD zur 1. Central European Conference on Logistics, Miskolc, Ungarn; 2010.

Schmidt, Thorsten; Wölker, Christoph: Mit richtiger Technik und Software zum Erfolg. In: Logistik heute, Jahrgang 25, 4/ 2003, S.26-27.

Schmidt, Thorsten; Wölker, Christoph: Intelligent Sortieren. In: Hebezeuge und Fördermittel, Jahrgang 43, 5/ 2003, S.214-215.

VDI 3619: Sortiersysteme für Stückgut. Berlin: Beuth-Verlag 1983. 